\title{
Prototipo automatizado para el llenado y tapado de botellas de plástico
}

\section{RESUMEN}

El presente artículo describe la implementación de un prototipo automatizado para el llenado y tapado de botellas de plástico, con la finalidad de incrementar el nivel de calidad de los productos así como la productividad en el envasado de las botellas. El prototipo ha sido desarrollado con actuadores neumáticos, sensores, electroválvulas, solenoides, motores y un controlador lógico programable (PLC S7-1200) así como también una rueda de ginebra para que este pueda controlar el proceso de manera automática, reduciendo los cuellos de botella y permitiendo un llenado uniforme. Para la implementación del proyecto, la parte mecánica se conectó con la parte neumática y eléctrica.

Palabras clave: Prototipo, proceso automatizado, rueda de ginebra.

AUTOMATED PROTOTYPE FOR THE FILLING AND CAPPING OF PLASTIC BOTTLES

\section{ABSTRACT}

This paper describes the implementation of an automated prototype for the filling and capping of plastic bottles, in order to increase the quality level of the products as well as the productivity in the bottling of the bottles. The prototype has been developed with pneumatic actuators, sensors, solenoid valves, solenoids, motors and a programmable logic controller (PLC S7-1200) as well as a gin wheel so that it can control the process automatically, reducing the bottlenecks And allowing uniform filling. For the implementation of the project, the mechanical part was connected to the pneumatic and electrical part.

Keywords: Prototype, automated process, geneva wheel.

\section{INTRODUCCIÓN}

Es necesario mencionar que el incremento de la demanda de agua embotellada en el mercado es cada vez mayor, por lo que la técnica del proceso de producción de agua debe mejorarse para reducir sus costos de producción. Según Hale (2010), el proceso de embotellado de botellas corresponde a una industria de procesos y las industrias relacionadas con la automatización son básicamente la industria manufacturera y la industria de procesos. Este proyecto fue realizado por el alumno Juber Gonzales Prado en el curso "Procesos de Manufactura Asistida por Computadora II", de la Escuela Profesional de Ingeniería Industrial de la Universidad Ricardo Palma.

El proyecto, si bien es cierto está enfocado al embotellado de agua, se puede extrapolar a cualquier otro líquido que requiera ser embotellado. El proyecto consta de cinco etapas cada una de las cuales se describe de manera concisa según lo implementado.

\section{Etapa 1: Alimentación de botellas}

Para comenzar este proceso se colocan las botellas de plástico vacías en la faja transportadora que es controlada por un motor de 220 VAC, HP 3/4, RPM 2850. La velocidad del motor fue calibrada dado que posee un embrague, esta faja soporta 5 botellas vacías. La faja transportadora de $60 \mathrm{cms}$. de largo está hecha de una estructura de metal y una correa articulada de PVC.

\section{Etapa 2: Llenado de botellas}

En el llenado de botellas se usó una matriz con 10 cavidades donde entran las botellas vacías. La matriz usa un movimiento rotativo paso a paso, esto se pudo realizar gracias a una rueda de ginebra que convierte un movimiento circular continuo en un movimiento circular intermitente. La Figura 1 muestra la matriz en donde se posicionan las botellas.

\footnotetext{
Doctor en Gestión Empresarial, docente de Ingeniería Industrial, URP.

E-mail: jose.velasquezc@urp.pe

2 Alumno de la Escuela de Ingeniería Industrial.

E-mail: juber.gonzales@urp.edu.pe
} 


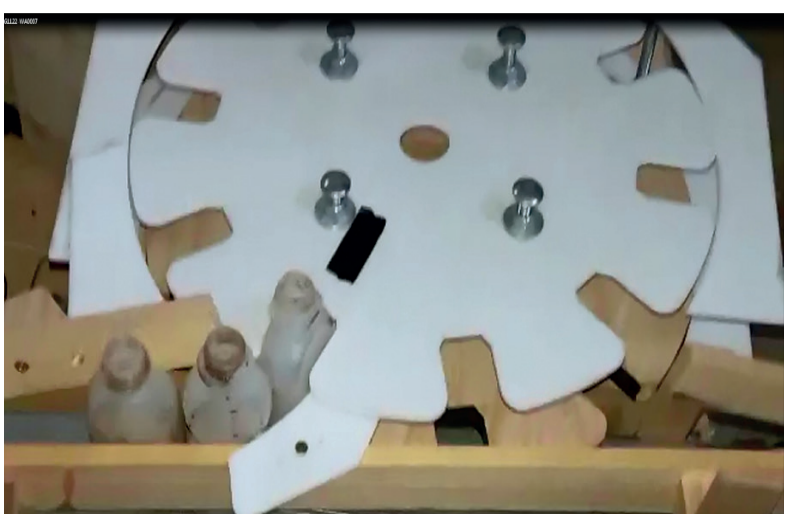

Fuente: Elaboración propia.

Figura 1. Matriz con 10 cavidades.

El movimiento circular continúo esta enlazado por una faja a un motor de paso.

Se empleó un motor de paso porque estos motores poseen más fuerza y precisión ya que tienen 2 o 4 bobinas en vez de una sola como la de un motor convencional. Normalmente este tipo de motor necesita de un driver para funcionar pero para este proyecto se conectó de una manera directa a un condensador y a un transformador de 12VAC. La Figura 2 muestra la parte mecánica de la rueda de ginebra.

Cuando la botella se sitúa debajo de la válvula solenoide un sensor óptico detecta la botella y hace que se detenga la matriz y en segundos la válvula solenoide se abre permitiendo el paso del líquido hacia la botella, el líquido es abastecido por un bidón conectado con mangueras a la válvula solenoide. La utilización principal de los sensores ópticos es como detectores de posición según Velásquez (2005).

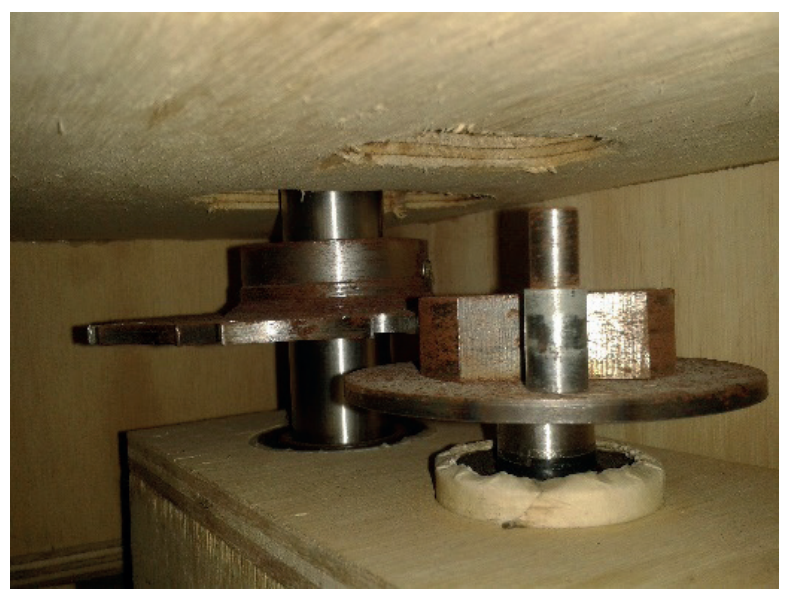

Fuente: Elaboración propia.

Figura 2. Parte mecánica de la rueda de ginebra.

\section{Etapa 3: Colocado de Tapas roscadas}

Una vez que la botella está llena pasara por debajo del dispensador de tapas. Para la implementación del proyecto se hizo un prototipo del dispensador en madera y cartón. La Figura 3 muestra el dispensador de tapas. Mientras la matriz gira, la botella captura la tapa y se posiciona en la boquilla de la botella plástica. Las tapas se colocan de manera manual una tras otra. El dispensador de tapas tiene una capacidad de 10 tapas.

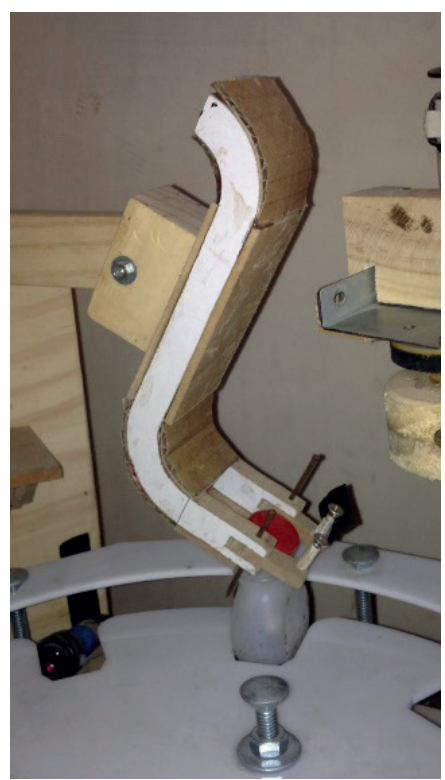

Fuente: Elaboración propia.

Figura 3. Dispensador de tapas.

\section{Etapa 4: Roscado de Tapa}

En esta etapa, la botella ya tiene puesta la tapa entonces mientras gira la matriz posicionara la botella debajo de un motor de 24 VDC al que se le fabricó un molde roscador (ver Figura 4) del tamaño de la tapa. Existe un sensor óptico detecta la botella y hace que la matriz se detenga para poner en acción el cilindro de doble efecto que posee sensores magnéticos en sus extremos y que está conectado a una electroválvula 5/2 monoestable de 24VDC.

El motor desciende por medio de un cilindro neumático y rosca la tapa. La neumática para Salvador (2003) es la tecnología que utiliza el aire comprimido como medio transmisor de energía y engloba el conjunto de técnicas para su transmisión, control y regulación. En esta etapa es importante el diseño que tiene la botella y la cavidad de la matriz, ya que al momento de roscar la tapa, la botella también puede girar. Para evitar este inconveniente se eligieron botellas de forma rectangular. 


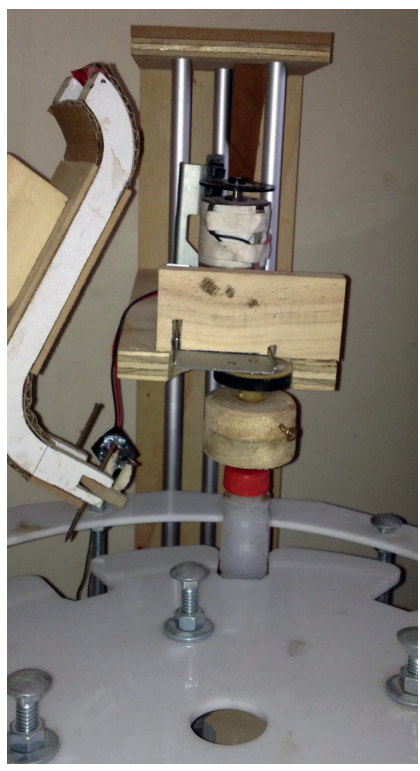

Fuente: Elaboración propia.

\section{Etapa5: Distribución de botellas llenas}

En la etapa final del proceso donde las botellas ya están llenas y tapadas y que pasaran a un proceso de etiquetado, el cual se implementará en un próximo proyecto.

\section{Diagrama de análisis de los procesos (DAP) ac- tual del embotellado manual}

Esta herramienta posibilita un conocimiento que sirva de base para la investigación donde se reconocerán los tiempos del proceso de embotellado de agua en envases de plástico de $500 \mathrm{ml}$ de capacidad. El Diagrama de Análisis del Proceso (DAP) actual se muestra en la Figura 5.

Figura 4. Roscado de la tapas.

DAP del Proceso de embotellado manual

\begin{tabular}{|c|c|c|c|c|c|c|c|c|}
\hline \multicolumn{9}{|c|}{ Proceso de envasado de botellas } \\
\hline Ubicación & & \multicolumn{5}{|c|}{ Actividad } & \multicolumn{2}{|c|}{ Metodo actual } \\
\hline \multirow[t]{2}{*}{ Actividad } & \multirow{2}{*}{$\begin{array}{l}\text { Produccion de envase } \\
\text { y enrroscado }\end{array}$} & \multicolumn{4}{|c|}{ Operación } & $\bigcirc$ & \multicolumn{2}{|c|}{4} \\
\hline & & \multicolumn{4}{|c|}{ Transporte } & $\Rightarrow$ & \multicolumn{2}{|c|}{1} \\
\hline Fecha & & \multicolumn{4}{|c|}{ Demora } & $D$ & \multicolumn{2}{|c|}{0} \\
\hline Operador & Analista & \multicolumn{4}{|c|}{ Inspeccion } & $\square$ & \multicolumn{2}{|c|}{2} \\
\hline \multirow{3}{*}{\multicolumn{2}{|c|}{ Comentarios: }} & \multicolumn{4}{|c|}{ Almacen } & $\nabla$ & \multicolumn{2}{|c|}{1} \\
\hline & & \multicolumn{5}{|c|}{ Tiempo (seg) } & \multicolumn{2}{|c|}{15} \\
\hline & & \multicolumn{5}{|c|}{ Distancia } & & \\
\hline \multirow{2}{*}{\multicolumn{2}{|c|}{ Descripcion de la actividad }} & \multicolumn{5}{|c|}{ Simbolos } & \multirow{2}{*}{$\begin{array}{l}\text { Tiempo } \\
\text { (seg) }\end{array}$} & \multirow{2}{*}{$\begin{array}{c}\text { Distancia } \\
\text { (mts) }\end{array}$} \\
\hline & & 0 & $\Rightarrow$ & $D$ & $\square$ & $\nabla$ & & \\
\hline Alimentacion de botellas & & - & & & & & 2 & \\
\hline Llenado de las botellas & & $\bullet$ & & & & & 4 & \\
\hline Inspeccion & & & & & $\bullet$ & & 2 & \\
\hline Colocado de tapas & & $\bullet$ & & & & & 2 & \\
\hline Roscado de tapa & & $\bullet$ & & & & & 2 & \\
\hline Inspeccion & & & & & - & & 1 & \\
\hline Distribucion de la botella & & & • & & & & 2 & \\
\hline Almacen & & & & & & $\bullet$ & & \\
\hline
\end{tabular}

Fuente: Elaboración propia.

Figura 5. DAP del Proceso de embotellado manual. 


\section{Actividades realizadas}

A continuación se presenta un listado de actividades realizadas para la culminación del proyecto con su respectiva duración en días. La tabla 1 detalla las actividades realizadas para la implementación del proyecto.

Tabla 1. Actividades para la implementación del proyecto.

\begin{tabular}{lc}
\hline Nombre de actividad & Duración \\
\hline Diseño Preliminar & 8 días \\
Compra de materiales & 4 días \\
Armado y Ensamblado de estructura & 20 días \\
Instalación de Sensores & 3 días \\
Instalación de cilindros y solenoides & 2 días \\
Cableado del PLC & 4 días \\
Programación del PLC & 8 días \\
Pruebas Iniciales & 3 días \\
Prueba Final & 3 días \\
\hline
\end{tabular}

Fuente: Elaboración propia.

\section{Diseño (SolidWorks)}

El diseño del proyecto se realizó en un software CAD teniendo en cuenta las especificaciones de tamaño de envases y características de los 3 motores y cilindro neumático. La Figura 6 muestra el diseño realizado en el software CAD.

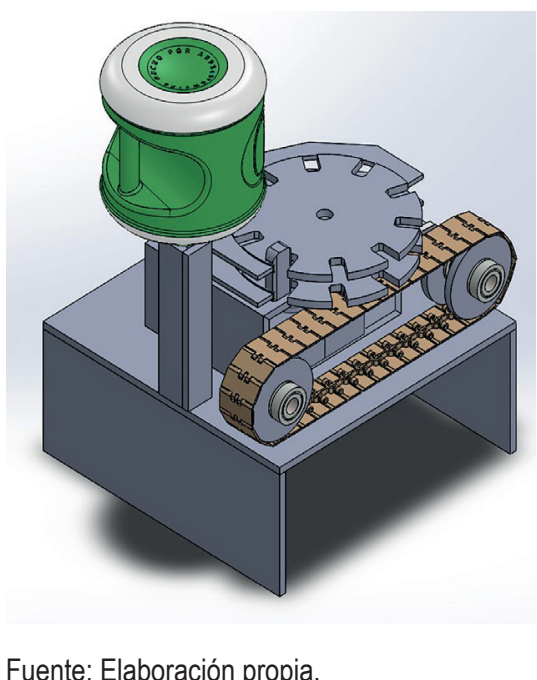

Figura 6. Diseño del prototipo automatizado.

\section{Diagrama de análisis de los procesos (DAP) ac- tual del embotellado automatizado.}

El DAP del Proceso Automatizado para el llenado y tapado de botellas de plástico se muestra en la Figura 7.

\begin{tabular}{|c|c|c|c|c|c|c|c|c|}
\hline \multicolumn{9}{|c|}{ Proceso de envasado de botellas } \\
\hline Ubicación & & \multicolumn{5}{|c|}{ Actividad } & \multicolumn{2}{|c|}{ Metodo actual } \\
\hline \multirow[t]{2}{*}{ Actividad } & \multirow{2}{*}{$\begin{array}{l}\text { Produccion de envase } \\
\text { y enrroscado }\end{array}$} & \multicolumn{4}{|c|}{ Operación } & $\bigcirc$ & \multicolumn{2}{|c|}{4} \\
\hline & & \multicolumn{4}{|c|}{ Transporte } & $\Rightarrow$ & \multicolumn{2}{|c|}{1} \\
\hline Fecha & & \multicolumn{4}{|c|}{ Demora } & $D$ & \multicolumn{2}{|c|}{0} \\
\hline Operador & Analista & \multicolumn{4}{|c|}{ Inspeccion } & $\square$ & \multicolumn{2}{|c|}{1} \\
\hline \multirow{3}{*}{\multicolumn{2}{|c|}{ Comentarios: }} & \multicolumn{4}{|c|}{ Almacen } & $\nabla$ & \multicolumn{2}{|c|}{1} \\
\hline & & \multicolumn{5}{|c|}{ Tiempo (seg) } & \multicolumn{2}{|c|}{11} \\
\hline & & \multicolumn{5}{|c|}{ Distancia } & & \\
\hline \multirow{2}{*}{\multicolumn{2}{|c|}{ Descripcion de la actividad }} & \multicolumn{5}{|c|}{ Simbolos } & \multirow{2}{*}{$\begin{array}{l}\text { Tiempo } \\
\text { (seg) }\end{array}$} & \multirow{2}{*}{$\begin{array}{c}\text { Distancia } \\
\text { (mts) }\end{array}$} \\
\hline & & 0 & $\Rightarrow$ & $D$ & $\square$ & $\nabla$ & & \\
\hline Alimentacion de botellas & & $\bullet$ & & & & & 2 & \\
\hline Llenado de las botellas & & $\bullet$ & & & & & 2 & \\
\hline Colocado de tapas & & $\bullet$ & & & & & 1 & \\
\hline Roscado de tapa & & $\bullet$ & & & & & 2 & \\
\hline Inspeccion & & & & & $\bullet$ & & 2 & \\
\hline Distribucion de la botella & & & $\bullet$ & & & & 2 & \\
\hline Almacen & & & & & & $\bullet$ & & \\
\hline
\end{tabular}

Fuente: Elaboración propia.

Figura 7. DAP del Proceso de embotellado automático. 


\section{Programación del PLC S7-1200}

Se necesitó programar el PLC para la automatización del prototipo de embotellado de botellas plásticas. La Figura 8 muestra a detalle la programación que se realizó para que el proceso funcione
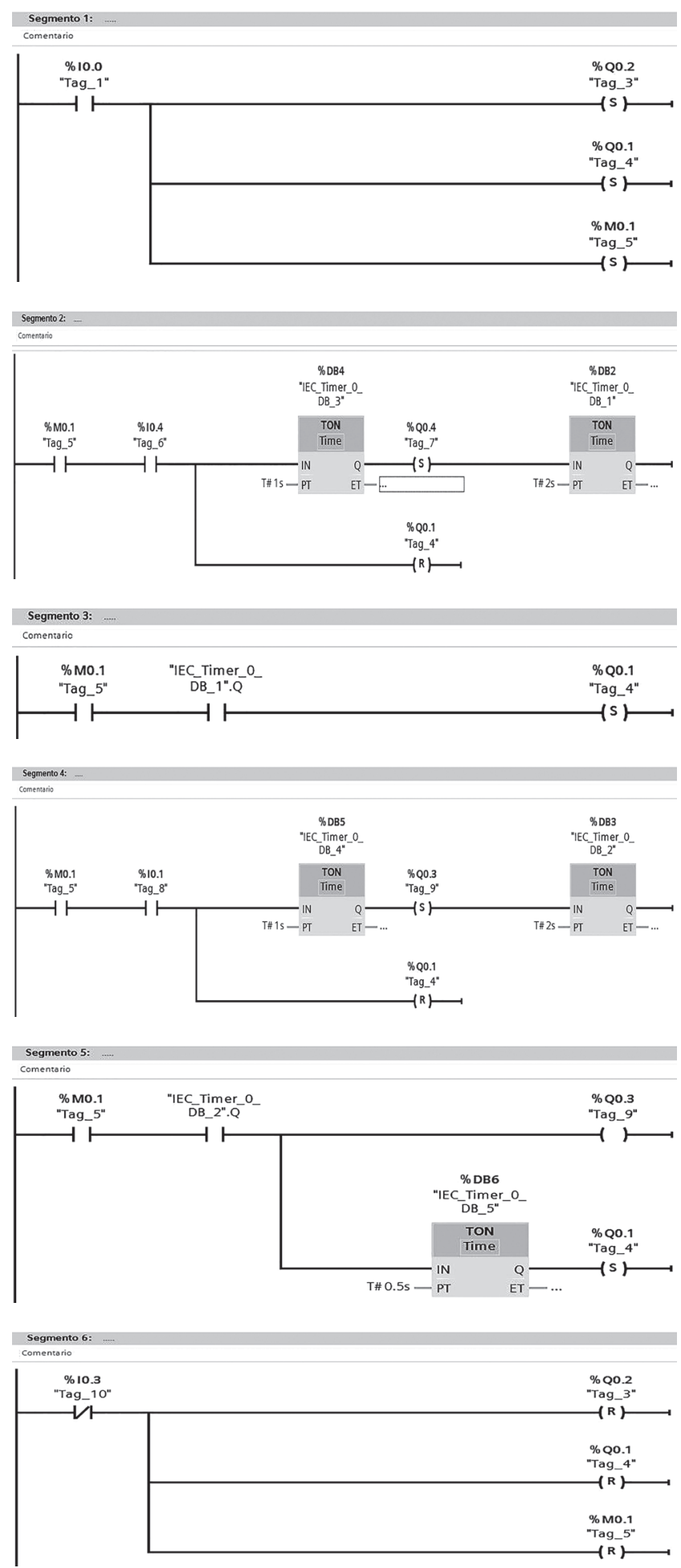

Fuente: Elaboración propia.

Figura 8. Programación del PLC S7-1200. de manera automática. Cada una de las etapas del proceso son controladas por un PLC según Velásquez (2009). El PLC empleado es de marca Siemens modelo S7-1200. A continuación se detalla su programación.

\section{Costos del Proyecto}

Para la implementación del prototipo de embotellado de botellas plásticas se tuvieron los siguientes costos (Tabla 2):

Tabla 2. Costo del proyecto.

\begin{tabular}{clr}
\hline cant & \multicolumn{1}{c}{ Descripción del item } & Total (S/.) \\
\hline 1 & Estructura del proyecto & S/. 300 \\
1 & Cilindro Neumático de doble efecto & S/. 70 \\
1 & Electroválvulas 5/2 & $\mathrm{S} / .50$ \\
1 & Válvula Solenoide & $\mathrm{S} / .80$ \\
2 & Sensor Óptico & $\mathrm{S} / .110$ \\
1 & Faja transportadora Industrial & $\mathrm{S} / .150$ \\
2 & Sensores magnéticos & $\mathrm{S} / .200$ \\
1 & Motor Paso a Paso & $\mathrm{S} / .15$ \\
1 & Motor 24VDC & $\mathrm{S} / .10$ \\
1 & Motor HOH SING de 220 VAC & $\mathrm{S} / .200$ \\
1 & PLC SIEMENS S7 1200 & $\mathrm{S} / .600$ \\
1 & Condensador 1100 uf & $\mathrm{S} / .10$ \\
4 & cables cocodrilos & $\mathrm{S} / .6$ \\
1 & Relé & $\mathrm{S} / .10$ \\
1 & Transformador 12VAC & $\mathrm{S} / .20$ \\
3 & Pulsadores & $\mathrm{S} / .15$ \\
1 & Reductor de Velocidad & $\mathrm{S} / .20$ \\
\hline & & $\mathrm{S} / .1,866$ \\
\hline & & TOTAL
\end{tabular}

Fuente: Elaboración propia.

Según los DAPs presentados anteriormente, se pueden evidenciar las siguientes ventajas del proyecto:

- Reducción del tiempo de embotellado en $36 \%$

- Satisfacción garantizada en el cliente por conceptos de calidad del producto, debido a que el contenido de los envases siempre tendrá el mismo volumen.

- Disminución en $90 \%$ las devoluciones de botellas por parte del consumidor final

- Posibilidad de ingreso a nuevos mercados, debido a las mejores condiciones de embotellado. 


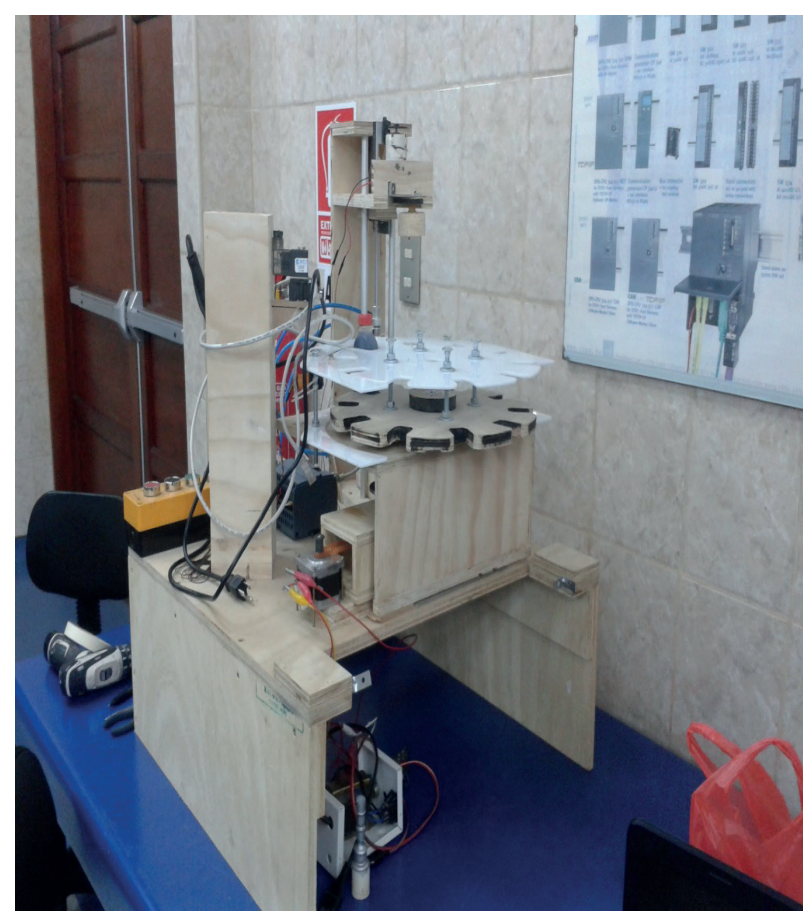

Fuente: Elaboración propia.

Figura 9. Foto de la implementación del proyecto.

\section{CONCLUSIONES}

- La automatización de procesos mejora la producción y productividad de las empresas, para este proyecto de embotellado en particular la producción se ve incrementada en $36 \%$.
- El proceso al estar programado mediante un controlador lógico programable (PLC), ejecuta cada acción de manera automática, lo que permite una producción continua.

- Se evitan reprocesos por un mal llenado o una inadecuada operación de roscado.

- Se reducen los tiempos así como también las actividades del proceso. Esto será beneficioso en grandes producciones donde la fatiga del operario será menor y la productividad aumentara.

- Al comienzo la inversión es grande pero esta se recuperara con un mayor número de producción en donde el costo por botella será menor.

\section{REFERENCIAS BIBLIOGRÁFICAS}

[1] Hale, M. (2010). Automation Made Easy. United States of America: International Society of Automation (ISA).

[2] Salvador, J. (2003). Instalaciones neumáticas. Barcelona.: Editorial UOC.

[3] Velásquez J. (2005). Los sensores en la producción. Revista de la Facultad de Ingeniería de la Universidad Ricardo Palma, 112-116.

[4] Velásquez J. (2009). Implementación de un prototipo de una dispensadora y envasadora de chocolates. Industrial Data. Industrial Data 12(2), 95-99. 